\title{
On the Squeezed Number States and their Phase Space Representations
}

\author{
L. Albano ${ }^{1}$, D.F.Mundarain ${ }^{1}$ and J. Stephany ${ }^{1,2}$ \\ ${ }^{1}$ Universidad Simón Bolívar,Departamento de Física,Apartado Postal 89000, \\ Caracas 1080-A, Venezuela. \\ ${ }^{2}$ Abdus Salam International Centre for Theoretical Physics, Strada Costiera, \\ 11, 34014 Trieste, Italy. \\ e-mail: lalbano@fis.usb.ve,dmundara@fis.usb.ve,stephany@usb.ve
}

\begin{abstract}
We compute the photon number distribution, the $Q(\alpha)$ distribution function and the wave functions in the momentum and position representation for a single mode squeezed number state using generating functions which allow to obtain any matrix element in the squeezed number state representation from the matrix elements in the squeezed coherent state representation. For highly squeezed number states we discuss the previously unnoted oscillations which appear in the $Q(\alpha)$ function. We also note that these oscillations can be related to the photon-number distribution oscillations and to the momentum representation of the wave function.
\end{abstract}

UNIVERSIDAD SIMON BOLIVAR 


\section{Introduction}

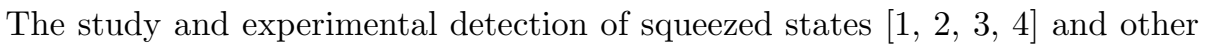
non classical states of optical systems, is an interesting issue, from both the fundamental and the technological point of view. On one hand, disentangling the properties of intrinsically quantum states of light enhances our understanding of the behavior and interactions of photons, and improves our knowledge of Quantum Electrodynamics. On the other hand, the use of light states with reduced quantum fluctuations in one of the conjugate quadratures is at the heart of attractive proposals for the detection of very weak signals 5 , 6 .

In this article we are concerned with the study of the squeezed number states and their phase space representations. These states emerged in the original analysis of Yuen [1] of the Two Photon Coherent States and have received since then a moderate amount of attention in the literature [1, 8, 9, 10, 11, 12]. They appear promissory for discussing the departure from the semiclassical regime in the context of quantum optics and for this reason were chosen for this study, but they also may have a practical interest on their own [12].

The one mode squeezed number states are obtained by applying the squeeze operator to the Fock states. As we discuss below, their wave functions in the position and momentum like quadratures are obtained by literally compressing or stretching the corresponding wave functions of the Fock states. An important aspect which holds our attention through this work is that they present oscillations in the photon number distribution as have been reported in Refs. [7. 8, 9]. These oscillations were first observed in the photon number distributions of squeezed states [13] which are equivalent to the Two-Photon Coherent States, and present several interesting properties. In a series of papers, [13, 14, 15, 16, 17], these oscillations were explained as the result of the interference of different contributions to probability amplitudes. These contributions were interpreted as the overlapping of the regions assigned to each quantum state on the phase space. For the squeezed number states oscillations, a closely related discussion was sketched in [7, 8]. This treatment which is physically very appealing is based on the semiclassical representation of states as bounded areas in phase space obtained with the help of Wigner distributions [19]. In Ref. 16] for the case of the squeezed states this area was fixed by weighting the Bohr-Sommerfeld rings with the Wigner-Cohen [20] distribution function. The same prescription was followed in Refs. [7, 8, 9] for the squeezed number states. In Ref [18], using the WKB method, a more general framework for the use of this picture was established.

In this paper we study the properties of the squeezed number states with the help of various representations. We compute explicitly the probability amplitudes and distributions of the squeezed number states in the Fock, position and momentum representations. We also compute the $Q(\alpha)$ distribution which has the advantage of being a true probability distribution which can be interpreted as the probability of a simultaneous measurement of position and momentum

within some region of the phase space. For high squeezing, the $Q(\alpha)$ function of a squeezed number state presents intrinsic oscillations in the space of the com- 
plex labels $\alpha$. These oscillations were not discussed previously in the literature and can be correlated with those in the momentum and Fock representations.

Our computations are done using a generating function technique which can be applied quite generally to compute expectation values of arbitrary observables in different representations.

\section{Two photon coherent states and squeezed num- ber states}

We consider here a single mode of the radiation field described in terms of the creation and annihilation operators $a^{\dagger}, a$, the number operator $\hat{N}_{a}=a^{\dagger} a$, and the momentum-like and position-like quadratures $\hat{p}=\frac{1}{i \sqrt{2}}\left(a-a^{\dagger}\right)$ and $\hat{q}=$ $\frac{1}{\sqrt{2}}\left(a+a^{\dagger}\right)$. Number (Fock) states $|n\rangle$ and coherent states $|\beta\rangle$ are the eigenstates of $\hat{N}_{a}$ and $a$ with eigenvalues $n$ and $\beta$ respectively. Coherent states may also be characterized as displaced vacuum states and are minimal uncertainty states satisfying $\Delta \hat{q}^{2}=\Delta \hat{p}^{2}=\frac{1}{2}$. We are working in units such that $h=2 \pi$. In the number state representation they are written as:

$$
|\beta\rangle=e^{-|\beta|^{2} / 2} \sum_{n} \frac{\beta^{n}}{\sqrt{n !}}|n\rangle .
$$

Squeezed states and squeezed number states may be obtained directly from $|\beta\rangle$ and $|n\rangle$ by the application of the squeeze operator. This operator depends on a complex parameter $\xi \equiv r e^{-i \phi}$. For simplicity we restrict our computation to the case of real $\xi$. We define $S(r)$ as the squeeze operator, and therefore

$$
\begin{array}{r}
|\beta, r\rangle=S(r)|\beta\rangle \\
|m, r\rangle=S(r)|m\rangle
\end{array}
$$

where $S(r)$ is given by

$$
S(r)=\exp \left(\frac{1}{2} r\left(a^{\dagger}\right)^{2}-\frac{1}{2} r a^{2}\right) .
$$

It is also useful to define a transformed annihilation operator $b$ as:

$$
b=S a S^{\dagger}=\cosh (r) a+\sinh (r) a^{\dagger}
$$

and then, one has

$$
b|\beta, r\rangle=\beta|\beta, r\rangle
$$

and

$$
\hat{N}_{b}|m, r\rangle=b^{\dagger} b|m, r\rangle=m|m, r\rangle .
$$

Given the linear nature of the transformation on $a$ (5), one readily computes the coherent states and Fock states amplitudes for the squeezed states [1] , 


$$
\begin{array}{r}
\langle\alpha \mid \beta, r\rangle=\frac{1}{\cosh ^{1 / 2}(r)} \exp \left(-\frac{1}{2}|\alpha|^{2}-\frac{1}{2}|\beta|^{2}-\frac{\tanh (r)}{2} \alpha^{* 2}\right. \\
\left.+\frac{\tanh (r)}{2} \beta^{2}+\frac{1}{\cosh (r)} \alpha^{*} \beta\right) \\
\langle n \mid \beta, r\rangle=\frac{\tanh ^{n / 2}(r)}{\left(2^{n} n ! \cosh (r)\right)^{1 / 2}} \mathrm{H}_{n}\left(\frac{\beta}{(2 \sinh (r) \cosh (r))^{1 / 2}}\right) \\
\times \exp \left(-\frac{1}{2}|\beta|^{2}+\frac{1}{2} \tanh (r) \beta^{2}\right)
\end{array}
$$

where $\mathrm{H}_{n}$ is the $n$th order Hermite polynomial.

For $\beta \in \mathbb{R}$, the wave functions in $q$ and $p$ are then given by:

$$
\begin{gathered}
\langle q \mid \beta, \xi\rangle=\left(2 \pi \Delta^{2} q\right)^{-1 / 4} \exp \left(-\frac{\left(q-q_{0}\right)^{2}}{4 \Delta^{2} q}\right) \\
\langle p \mid \beta, \xi\rangle=\left(\frac{2 \Delta^{2} q}{\pi}\right)^{1 / 4} \exp \left(-p^{2} \Delta^{2} q-i p q_{0}\right),
\end{gathered}
$$

with $q_{0}=\sqrt{2} e^{r} \beta, \Delta^{2} q=e^{-2 r} / 2$ and $\Delta^{2} p=e^{2 r} / 2$.

\section{Representations of the squeezed number states}

To discuss the properties of the squeezed number states, we observe that for any operator $\hat{R}$ and any state $|\psi\rangle$, the completeness of the Fock states and Eq. (1) allow one to write,

$$
\langle\psi|\hat{R}| \beta, r\rangle=\sum_{m}\langle\psi|\hat{R} S(r)| m\rangle\langle m \mid \beta\rangle=e^{-|\beta|^{2} / 2} \sum_{m} \frac{\beta^{m}\langle\psi|\hat{R}| m, r\rangle}{(m !)^{1 / 2}} .
$$

This provides a generating function for the matrix element $\langle\psi|\hat{R}| m, r\rangle$ which can then be obtained as,

$$
\langle\psi|\hat{R}| m, r\rangle=\frac{1}{(m !)^{1 / 2}}\left[\frac{\partial^{m}}{\partial \beta^{m}}\left(e^{|\beta|^{2} / 2}\langle\psi|\hat{R}| \beta, r\rangle\right)\right]_{\beta=0} .
$$

For example, if $\hat{R}=U(t)$ is the evolution operator, and $|\psi\rangle=|k\rangle$ with $\{|k\rangle\}$ being any complete basis of the radiation states, one can compute in this form the time dependent probability amplitudes in the $\{|k\rangle\}$ representation. For $t=0$ we get the probability amplitudes of stationary squeezed number states.

Generalizing the same idea, we also note that

$$
\left\langle\alpha_{g}, r|\hat{R}| \beta_{g}, r\right\rangle=e^{-1 / 2|\alpha|^{2}} e^{-1 / 2|\beta|^{2}} \sum_{n} \sum_{m} \frac{\alpha^{* n}}{(n !)^{1 / 2}} \frac{\beta^{m}}{(m !)^{1 / 2}}\langle n, r|\hat{R}| m, r\rangle
$$


so that we can write,

$$
\langle n, r|\hat{R}| m, r\rangle=\left.\frac{1}{(n ! m !)^{1 / 2}} \frac{\partial^{n}}{\partial \alpha^{* n}} \frac{\partial^{m}}{\partial \beta^{m}}\left[e^{1 / 2|\alpha|^{2}} e^{1 / 2|\beta|^{2}}\left\langle\alpha_{g}, r|\hat{R}| \beta_{g}, r\right\rangle\right]\right|_{\alpha^{*}=0, \beta=0} .
$$

With this observation we can generate the probability distributions for the squeezed number states in the Fock, position, momentum, and coherent states representations. Taking $|\psi\rangle=|n\rangle$ and $\hat{R}=1$, the amplitude for the squeezed number states in the Fock states basis is,

$$
\begin{array}{r}
\langle n \mid m, r\rangle=\frac{1}{(m !)^{1 / 2}}\left[\frac{\partial^{m}}{\partial \beta^{m}}\left(e^{|\beta|^{2} / 2}\left\langle n \mid \beta_{g}, r\right\rangle\right)\right]_{\beta=0}= \\
\frac{(\tanh (r) / 2)^{n / 2}}{\sqrt{n ! m ! \cosh (r)}}\left[\frac{\partial^{m}}{\partial \beta^{m}} \mathrm{H}_{n}\left(\frac{\beta}{\sqrt{2 \sinh (r) \cosh (r)}}\right) e^{\frac{\beta^{2} \tanh (r)}{2}}\right]_{\beta=0} .
\end{array}
$$

Next we note that,

$$
\begin{aligned}
& {\left[\mathrm{H}_{n}^{(l)}\left(\frac{\beta}{(2 \sinh (r) \cosh (r))^{1 / 2}}\right)\right]_{\beta=0}=} \\
& \frac{2^{l}(-1)^{n-l / 2} n !(2 \sinh (r) \cosh (r))^{-l / 2}}{((n-l) / 2) !}
\end{aligned}
$$

and

$$
\left.\left(e^{\frac{\beta^{2} \tanh (r)}{2}}\right)^{(l)}\right|_{\beta=0}=\left\{\begin{array}{ll}
0 & l \text { odd } \\
\left(\frac{\tanh (r)}{2}\right)^{l / 2} \frac{l !}{(l / 2) !} & l \text { even }
\end{array} .\right.
$$

Using the Cauchy formula

$$
\{f(x) g(x)\}^{(m)}=\sum_{k=0}^{m} \frac{m !}{k !(m-k) !} f^{(m-k)}(x) g^{(k)}(x)
$$

we get,

$$
\begin{array}{r}
\langle n \mid m, r\rangle=\frac{(m ! n !)^{1 / 2}}{\cosh (r)^{\frac{(n+m+1)}{2}}} \sum_{k}^{\min (m, n)}\left(\frac{\sinh (r)}{2}\right)^{\frac{(n+m-2 k)}{2}} \frac{(-1)^{\frac{(n-k)}{2}}}{k !\left(\frac{m-k}{2}\right) !\left(\frac{n-k}{2}\right) !} \\
k=\left\{\begin{array}{ll}
0,2,4,6 \ldots & n, m \text { even } \\
1,3,5,7 \ldots & n, m \text { odd }
\end{array} .\right.
\end{array}
$$

This result is in agreement with the one reported in Ref. [7], which was obtained by the application of a normally ordered squeeze operator to a Fock state. The most salient features of this photon distribution are that it oscillates and that only the photon number states of the same parity of $m$ are represented in the expansion for $|m, r\rangle$. The latter reflects of course the quadratic dependence of $S(r)$ in $a$ and $a^{\dagger}$. The oscillations of the distribution $P_{n, m} \equiv|\langle n \mid m, r\rangle|^{2}$ for 
$m>1$ and $r$ sufficiently large have a fixed number of maxima; $m / 2+1$ when $m$ is even and $(m+1) / 2$ when $m$ is odd. These characteristics can be related to the structure of the $Q(\alpha)$ function, as we discuss below.

For the squeezed number states with $m=0$ (the squeezed vacuum) and $m=1$ the sum in equation 20 is just a single term:

$$
\begin{gathered}
|\langle n \mid 0, r\rangle|^{2}=\frac{n !}{\left(\left(\frac{n}{2}\right) !\right)^{2} 2^{n}} \frac{\tanh ^{n}(r)}{\cosh (r)} \\
n=0,2,4,6, \ldots . \\
|\langle n \mid 1, r\rangle|^{2}=\frac{n !}{\left(\left(\frac{n-1}{2}\right) !\right)^{2} 2^{n-1}} \frac{\tanh ^{n-1}(r)}{\cosh ^{3}(r)} . \\
n=1,3,5,7, \ldots . .
\end{gathered}
$$

As an example, we show in Figure (11) the photon number distribution for a squeezed number state with $m=7$ and $r=1$.4. The distribution $|\langle n \mid 7,1.40\rangle|^{2}$ has 4 maxima in $n$ located at 1,11,37 and 89 photons respectively.

The support of the distribution, and the oscillations, become wider for greater $r$. For the squeezed number state with $m=7$, this is illustrated by Figure (2). In this figure, we plot the positions of $n$ for the last three maxima as functions of $r$. These values shift to the right as $r$ grows. It is possible to observe a tendency for each of the maxima, which can be approximated by exponential functions .

Let us study the other representations of squeezed number states. Using (10) and (13) we have

$$
\langle q \mid m, r\rangle=\left.m !^{-1 / 2}(2 \pi)^{-1 / 4}\left(2^{-1 / 2} e^{-r}\right)^{-1 / 2} \frac{\partial^{m}}{\partial \beta^{m}}\left\{e^{\left(\frac{-\left(q-\sqrt{2} e^{r} \beta\right)^{2}}{2 e^{-2 r}}\right)} e^{\beta^{2} / 2}\right\}\right|_{\beta=0} .
$$

And using again the Cauchy formula we have

$$
\frac{\partial^{m}}{\partial \beta^{m}}\left\{e^{\left(\frac{-\left(q-\sqrt{2} e^{r} \beta\right)^{2}}{2 e^{-2 r}}\right)} e^{\beta^{2} / 2}\right\}=\sum_{k=0}^{m} \frac{m !}{(m-k) ! k !} \frac{\partial^{k}}{\partial \beta^{k}} e^{\left(\frac{-\left(q-\sqrt{2} e^{r} \beta\right)^{2}}{2 e^{-2 r}}\right)} \frac{\partial^{m-k}}{\partial \beta^{m-k}} e^{\beta^{2} / 2} .
$$

Evaluating at $\beta=0$ we obtain

$$
\begin{array}{r}
\langle q \mid m, r\rangle=\pi^{-1 / 4} e^{r / 2} e^{\left(-\frac{e^{2 r} q^{2}}{2}\right)} 2^{-m / 2} m !^{1 / 2} \sum_{k}^{m} \frac{2^{k / 2}}{k !((m-k) / 2) !} \mathrm{H}_{k}\left(\frac{e^{r} q}{2^{1 / 2}}\right) \\
k=\left\{\begin{array}{cc}
0,2,4,6 \ldots & m \text { even } \\
1,3,5,7 \ldots & m \text { odd }
\end{array}\right.
\end{array}
$$

In order to simplify the expression above we use the following identity of the Hermite polynomials,

$$
\frac{1}{m !} \mathrm{H}_{m}(x)=\sum_{k}^{m} \frac{2^{k / 2}}{k !((m-k) / 2) !} \mathrm{H}_{k}\left(\frac{x}{2^{1 / 2}}\right)
$$




$$
k=\left\{\begin{array}{cc}
0,2,4,6 \ldots & m \text { even } \\
1,3,5,7 \ldots & m \text { odd }
\end{array}\right.
$$

Finally, the amplitude (25) becomes:

$$
\langle q \mid m, r\rangle=\pi^{-1 / 4} e^{r / 2} e^{\left(-\frac{e^{2 r} q^{2}}{2}\right)} 2^{-m / 2} m !^{-1 / 2} \mathrm{H}_{m}\left(\frac{q}{e^{-r}}\right)
$$

Thus, the amplitude is that of a Fock state, depending on a squeezed quadrature variable $e^{r} q$. Moreover, the amplitude of a Fock state is given by the limit of (27) when $r \rightarrow 0$.

The representation of the squeezed number states in terms of the other quadrature follows along the same lines. We get

$$
\begin{array}{r}
\langle p \mid m, r\rangle=\frac{e^{-r / 2}}{m !^{1 / 2} \pi^{1 / 4}} e^{\left(-\frac{e^{-2 r_{p}}}{2}\right)} 2^{-m / 2} \sum_{k}^{m} \frac{m !\left(-2 i e^{-r} p\right)^{k}}{k !((m-k) / 2) !} \\
k=\left\{\begin{array}{cc}
0,2,4,6 \ldots & m \text { even } \\
1,3,5,7 \ldots & m \text { odd }
\end{array}\right.
\end{array}
$$

and

$$
\langle p \mid m, r\rangle=\frac{e^{-r / 2}}{m !^{1 / 2} \pi^{1 / 4}} e^{\left(-\frac{e^{-2 r} p^{2}}{2}\right)} 2^{-m / 2}(-i)^{m} \mathrm{H}_{m}\left(e^{-r} p\right)
$$

The amplitude depends on $e^{-r} p$ and, for $r>0,|\langle p \mid m, r\rangle|^{2}$ has a support that is $e^{r}$ wider than the support of a Fock state. The distribution has $m+1$ maxima and $m$ minima (which are also zeroes). In Figure (3) we show the momentum probability distribution for the $m=7$ squeezed number state with $r=1.4$. The oscillations in this distribution come from the Hermite polynomial. For large squeezing, these oscillations reappear in the $Q(\alpha)$ distribution function.

Finally, let us turn to the $Q(\alpha)$ function, which is given by the diagonal elements of the density matrix $\rho$ associated with the state in the coherent states basis.

For a squeezed number state one has,

$$
Q(\alpha)=\frac{1}{\pi}\langle\alpha|\rho| \alpha\rangle=\frac{1}{\pi}|\langle\alpha \mid m, \xi\rangle|^{2} .
$$

The function $Q(\alpha)$ gives the probability of being in a minimum dispersion state around an average position and momentum proportional to $\operatorname{Re}(\alpha)$ and $\operatorname{Im}(\alpha)$ [21]. Following the previous procedure and using (8), we have

$$
\langle\alpha \mid m, r\rangle=\left.\left(\frac{1}{m ! \cosh (r)}\right)^{1 / 2} e^{-1 / 2|\alpha|^{2}-\tanh (r) \alpha^{* 2} / 2} \frac{\partial^{m}}{\partial \beta^{m}} e^{\frac{\tanh (r)}{2} \beta^{2}+\frac{\alpha^{*}}{\cosh (r)} \beta}\right|_{\beta=0}
$$

which can be reduced to

$$
\langle\alpha \mid m, r\rangle=m !^{1 / 2} \cosh (r)^{-1 / 2} e^{-1 / 2|\alpha|^{2}-\tanh (r) \alpha^{* 2} / 2}
$$




$$
\begin{gathered}
\times \sum_{p=0}^{[m / 2]} \frac{2^{-p} \sinh ^{p}(r) \cosh ^{p-m}(r)}{(m-2 p) ! p !}\left(\alpha^{*}\right)^{m-2 p} \\
{[m / 2]=\left\{\begin{array}{lll}
m / 2 & m & \text { even } \\
\frac{m-1}{2} & m & \text { odd }
\end{array}\right.}
\end{gathered}
$$

The $Q(\alpha)$ function of a squeezed number state is, therefore:

$$
\begin{gathered}
Q(\alpha)=\frac{1}{\pi} m ! \cosh (r)^{-1} e^{-|\alpha|^{2}-\tanh (r)\left(\alpha^{* 2}+\alpha^{2}\right) / 2} \\
\times\left|\sum_{p=0}^{[m / 2]} \frac{2^{-p} \sinh ^{p}(r) \cosh ^{p-m}(r)}{(m-2 p) ! p !}\left(\alpha^{*}\right)^{m-2 p}\right|^{2} . \\
{[m / 2]=\left\{\begin{array}{lll}
m / 2 & m & \text { even } \\
\frac{m-1}{2} & m & \text { odd }
\end{array}\right.}
\end{gathered}
$$

The shape of $Q(\alpha)$ depends strongly on the squeezing parameter $r$, as illustrated by Figures (4), (5) and (6), (7); which show the Eq. (33) for $m=7$, $r=0.50$ and $r=1.40$.

For small squeezing, the $Q(\alpha)$ function is appreciably nonzero over an elliptical ring, with an eccentricity $e^{r}$. When $r=0$ this ring is circular, the $Q(\alpha)$ function being that of a Fock state. For greater squeezing, the $Q(\alpha)$ function shows prominent oscillations with a number of maxima which for the $m$ squeezed number state stabilize at $m+1$. As can be shown by direct numerical computation, the transition occurs at $r \simeq \frac{1}{2} \ln (m)$ which corresponds to $\sqrt{m} e^{-r}=1$. It is interesting to note that in the phase space analysis this behavior appears at the point where the inner boundary of the deformed ring touch itself and in this sense is also a consequence of interference in the phase space. Moreover it can be checked by direct numerical computation that this effect is registered only by the $Q$ function, and not by other interesting distributions like the Wigner or Wigner-Cohen functions.

The oscillations for $r>\frac{1}{2} \ln (m)$ occur along the axis of $\operatorname{Im}(\alpha)$ and are correlated to the oscillations of the momentum wave function. To show this explicitly observe that for high squeezing, the $Q(\alpha)$ function evaluated on the $\operatorname{Im}(\alpha)$ axis is proportional to the square of the momentum wave function:

$$
Q(\alpha=i \sqrt{2} p) \sim|\langle p \mid m, r\rangle|^{2}
$$

For illustration, consider the $m=7$ squeezed number state with $r=1.4$. The maxima in its momentum distribution (see Figure (3)) correspond to those of the $Q(\alpha)$ function (figure 6 ) in the $\operatorname{Im}(\alpha)$ axis.

In the same way, for large squeezing, the $Q(\alpha)$ oscillations are also correlated to the oscillations of the photon number distribution $|\langle n \mid m, r\rangle|^{2}$. Each maximum $\alpha_{\max }=i\left|\alpha_{\max }\right|\left(\left|\alpha_{\max }\right| \geq 1\right)$ of $Q$ is associated to a maximum $n_{\max }$ of $|\langle n \mid m, r\rangle|^{2}$, in such away that,

$$
n_{\max } \simeq\left|\alpha_{\max }\right|^{2} .
$$


In this form we can use the $Q$ function to obtain directly from the phase space a good picture of $P_{m}$ distribution of the state.

Finally, observe that the $Q$ distribution of a squeezed number state is given by ,

$$
Q(\alpha)=\frac{1}{\pi}|<\alpha| S|m>|^{2}=\frac{1}{\pi}|<m| S^{\dagger}|\alpha>|^{2} .
$$

and henceforth, the $Q(\alpha)$ function of a squeezed number state $|m, r\rangle$ is proportional to the photon number amplitude of a two photon coherent state $|\alpha, r\rangle=S^{\dagger} \mid \alpha>$. The oscillations still can be explained using the hypothesis of interference in phase space. To illustrate this point consider Figure (8) which shows the areas representing a Fock state and a pair of two photon coherent states $(|\alpha, r\rangle)$ with respectively real or imaginary $\alpha$. For $\alpha$ imaginary the overlapping area doubles and produces the interference effect in the $Q$ function. The photon statistical value of a two photon coherent state is computed using the method of overlapping areas in the phase space [22]. The result is,

$$
P_{m}=|<m| S^{\dagger}|\alpha=i y>|^{2}=\left|\sqrt{A m} e^{i \phi}+\sqrt{A m} e^{-i \phi}\right|^{2}
$$

with

$$
A_{m}=\frac{e^{-2\left(m+1 / 2-y^{2} / e^{2 r}\right) / e^{2 r}}}{\sqrt{2 \pi\left(m+1 / 2-y^{2} / e^{2 r}\right)}}
$$

and

$$
\begin{aligned}
\phi= & \left.(m+1 / 2) \arctan \left\{\sqrt{\left(m+1 / 2-y^{2} / e^{2 r} /\left(y^{2} / e^{2 r}\right.\right.}\right)\right\} \\
& \left.-x \sqrt{\left(m+1 / 2-y^{2} / e^{2 r} / e^{2 r}\right.}\right)-\pi / 4
\end{aligned}
$$

This match with the exact computation presented above as is shown in Figure (9) where we present respectively the $Q(\alpha=i y)$ function for the $m=7, r=1.4$ squeezed number state obtained using this approximation and the previous exact calculation. For sufficiently high values of $|\alpha|$ the WKB approximation on which is based the phase space computation no longer holds as evidenced in Figure (9).

\section{Conclusion}

We have computed the photon number distribution, the momentum like and position like wave functions, and the $Q(\alpha)$ function for squeezed number states and we have shown that each of them has characteristic oscillations which depend on the squeeze parameter $r$. For highly squeezed number states we observed that the oscillations in the different probability distributions are fixed in number and are in close correspondence between them. The $Q(\alpha)$ function in this case present a richer structure that for number states. The oscillations were also discussed within the semiclassical approximation using the phase space methods based in the WKB functions 18, 22. 
It should be also mentioned that the generating function formalism used throughout this work has been shown to be a straightforward and very valuable procedure for calculating probability amplitudes and matrix elements for any base obtained transforming number states by any arbitrarily chosen unitary operator.

\section{$5 \quad$ Acknowledgements}

We thank a referee for important observations on an earlier version of this article. This work was supported by DID-USB and by Grant N0 G-2001000712 of Fonacit.

\section{References}

[1] H. P. Yuen, Phys. Rev. A 13, 2226 (1976).

[2] D. F. Walls, Nature (London) 306, 141 (1983).

[3] L.-A. Wu, H. J. Kimble, J. L. Hall and H. Wu, Phys. Rev. Lett. 57, 2520 (1986).

[4] R. M. Shelby, M. D. Levenson, S. M. Perlmutter, R. G. De Voe and D. F. Walls, Phys. Rev. Lett. 57, 691 (1986).

[5] C. M. Caves, Phys. Rev. D 23, 1693 (1981).

[6] A. F. Pace, M. J. Collet and D. F. Walls, Phys. Rev. A 47, 3173 (1993).

[7] M. S. Kim, F. A. M. De Oliveira and P. L. Knight, Phys. Rev. A 40, 2494 (1989).

[8] M. S. Kim, F. A. M. De Oliveira and P. L. Knight, Optics Comm. 72, 99 (1989).

[9] Michael J. Gagen, Phys. Rev. A 51, 2715 (1995).

[10] M. M. Nieto, Los Alamos e-print archive quant-ph/9612050 (1996)

[11] K.B.Møller, T.G.Jørgensen and J.P.Dahl Phys. Rev. A 54, 5378 (1996).

[12] G. M. D'Ariano, Int. J. Mod. Phys. B 6, 1291 (1992).

[13] J. A. Wheeler, Lett. Math. Phys. 10, 201 (1985).

[14] W. Schleich and J. A. Wheeler, Nature 326, 574 (1987).

[15] H. Walther, W. Schleich and J. A. Wheeler, Foundations of Physics 18, 953 (1988).

[16] W. Schleich, D. F. Walls and J. A. Wheeler, Phys. Rev. A 38, 1177 (1988). 
[17] W. Schleich, J. P. Dowling, R. J. Horowicz and S. Varro, New Frontiers in Quantum Electrodynamics and Quantum Optics, ( ed. A. O. Barut ), 31 (Plenum,New York, 1990)

[18] J.P.Dowling, W.P.Schleich and J. A. Wheeler, Annalen der Physik 48, 423 (1991).

[19] M. Hillery, R. F. O'Conell,M. O. Scully and E. P. Wigner, Phys. Rep. 106, 121 (1984).

[20] L. Cohen, Frontiers of Nonequilibrium Statistical Physics (eds. G. T. Mooore \& M. O. Scully) 97 ( Plenum, New York, 1986).

[21] G. J. Milburn, Squeezed and nonclassical light, (eds. P. Tombesi and E. R. Pike), 151 ( Plenum, New York, 1989).

[22] Wolfgang P. Schleich, Quantum Optics in Phase Space, Chapter 4,WileyVch, Berlin, (2001). 


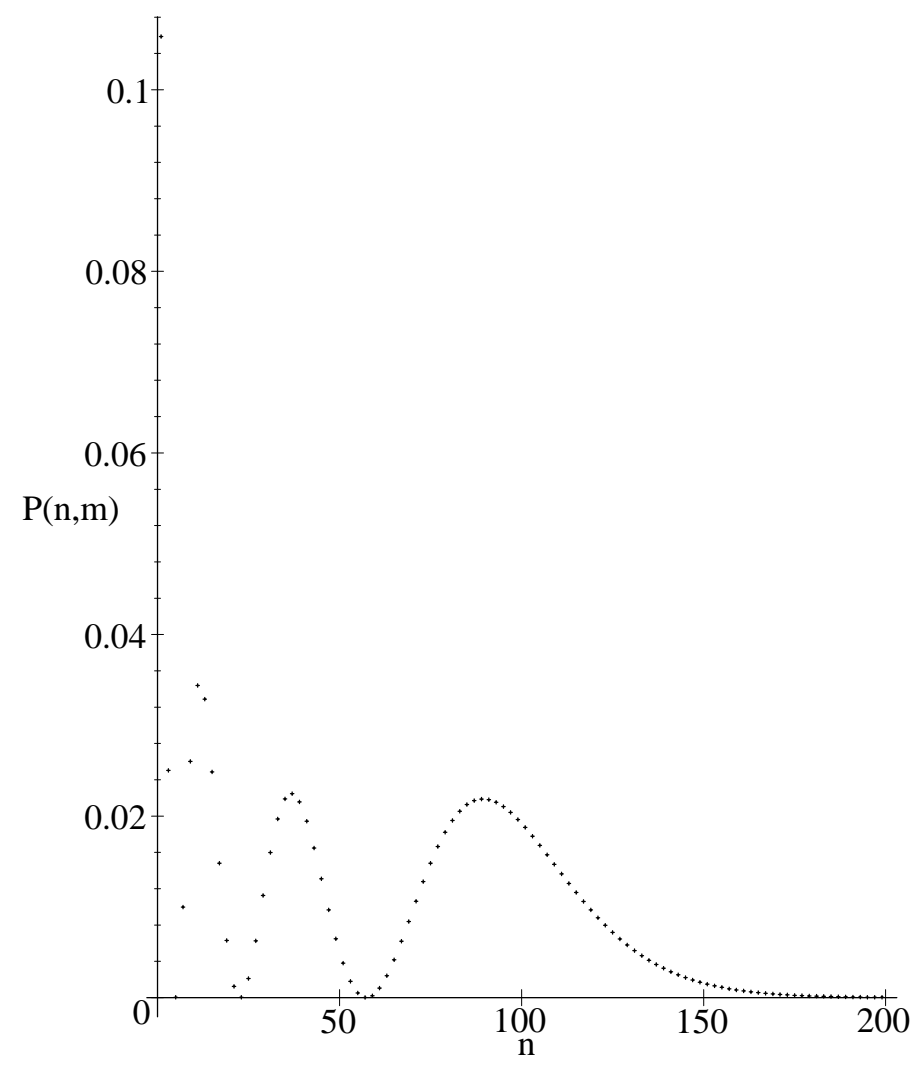

Figure 1: Photon number distribution for the squeezed number states with $m=7$ and $r=1.40,|\langle n \mid 7,1.40\rangle|^{2}$. 


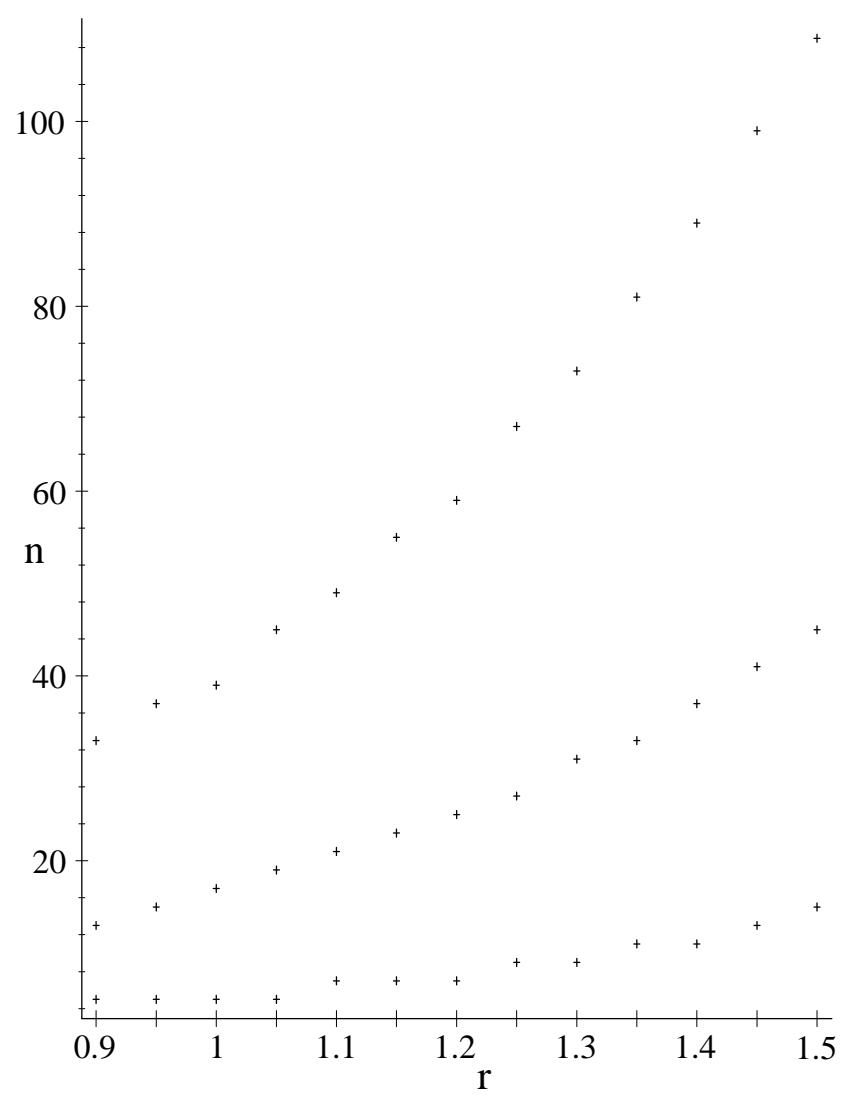

Figure 2: Position $n$ of the maxima for the photon number distribution $|\langle n \mid 7, r\rangle|^{2}$ of a squeezed number states, versus $r$ 


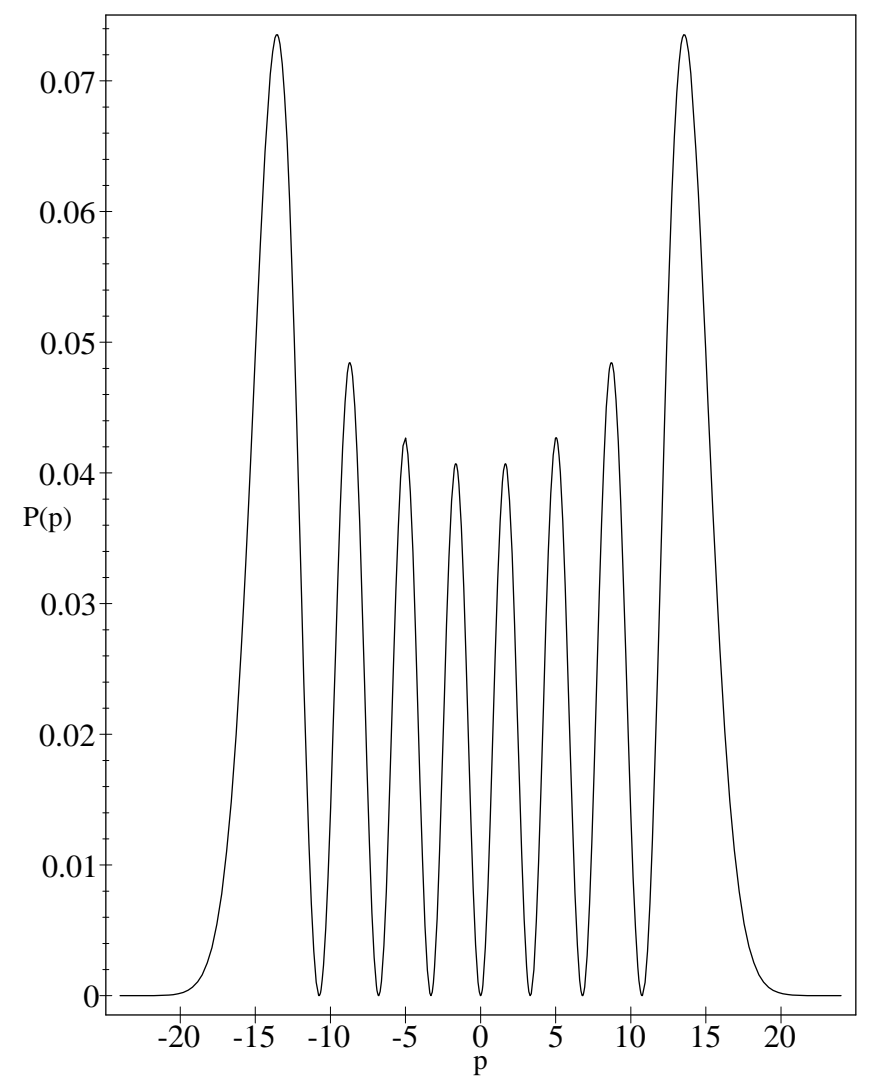

Figure 3: Momentum probability distribution $|\langle p \mid m, r\rangle|^{2}$ for the $m=7$ squeezed number state with $r=1.4$. 


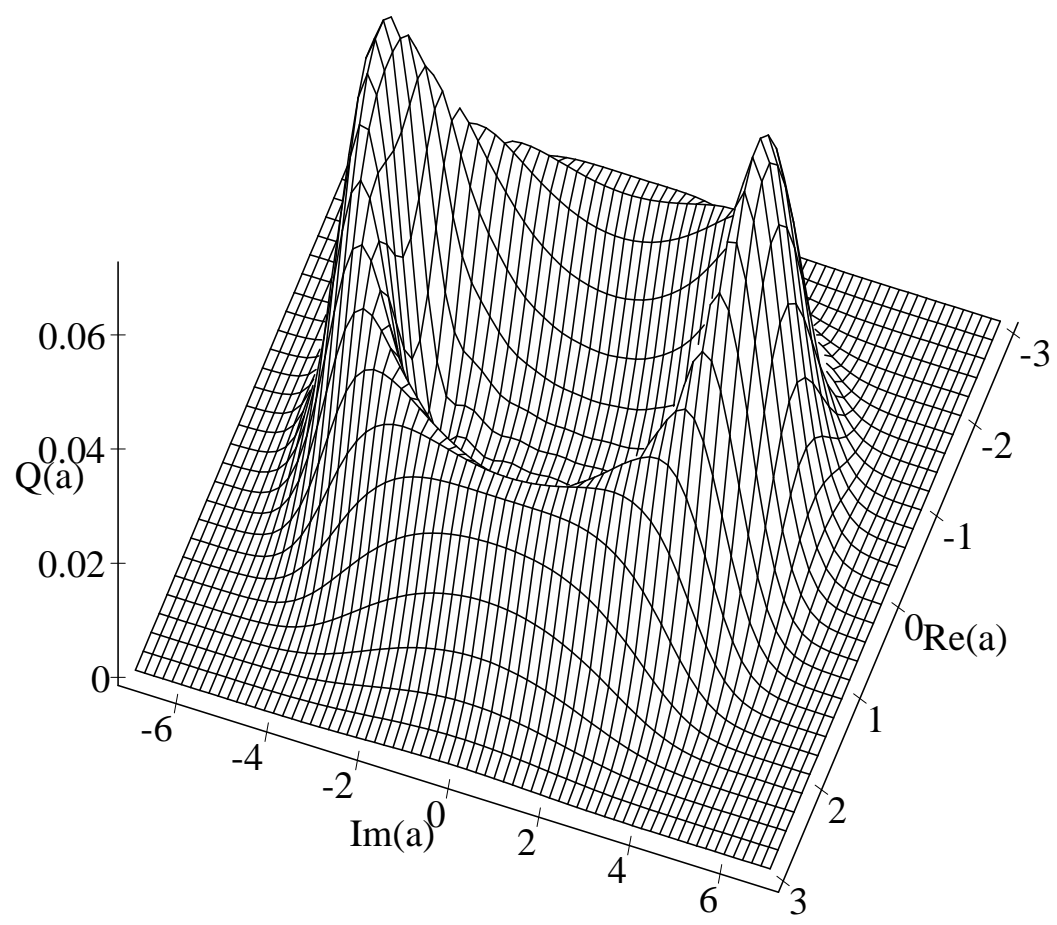

Figure 4: $Q(\alpha)$ function of the $m=7$ squeezed number state with $r=0.50$ 


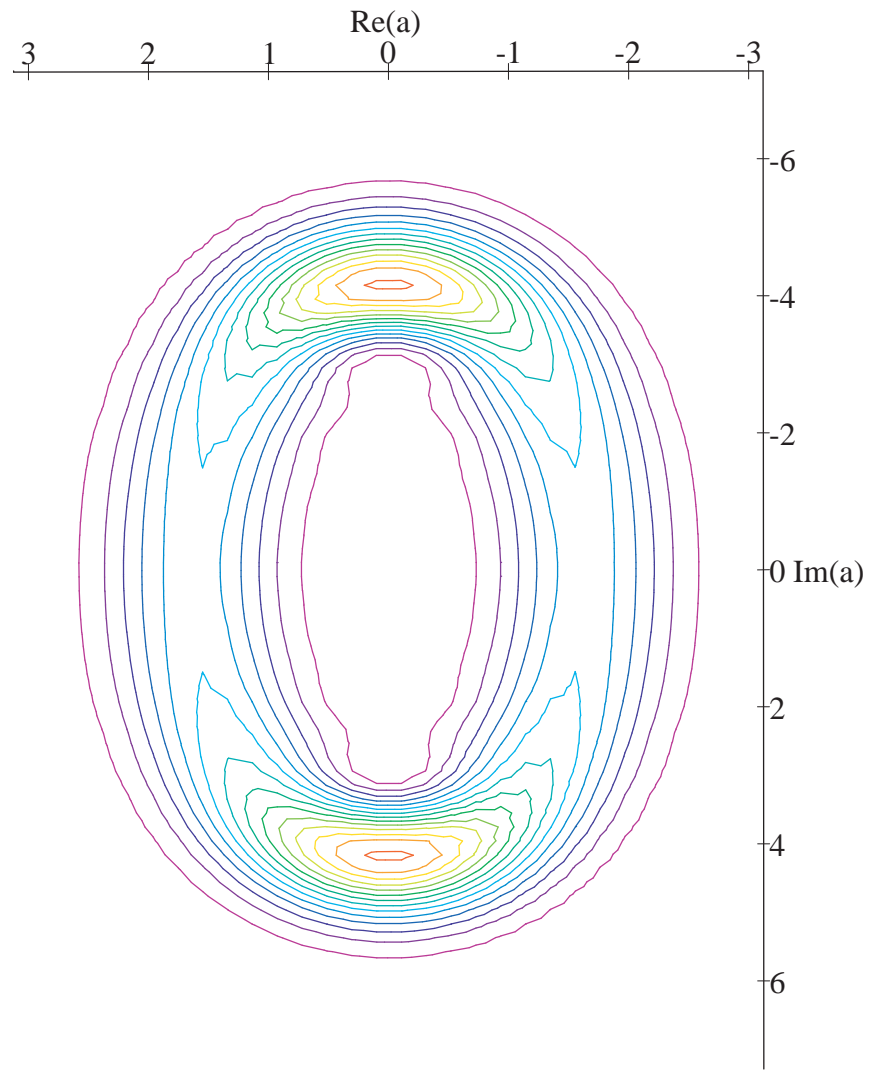

Figure 5: Contour graphic of the $Q(\alpha)$ function of the $m=7$ squeezed number state with $r=0.50$ 


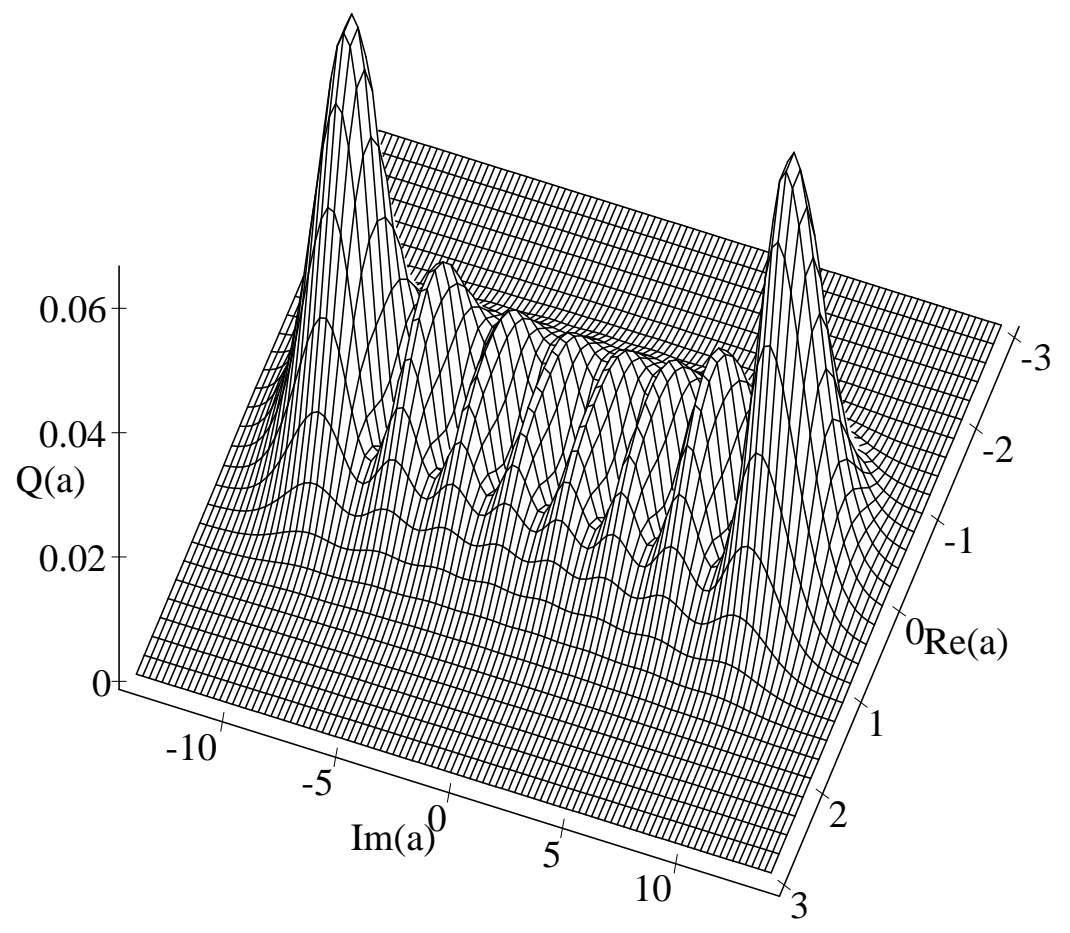

Figure 6: $Q(\alpha)$ function of the $m=7$ squeezed number state with $r=1.40$ 


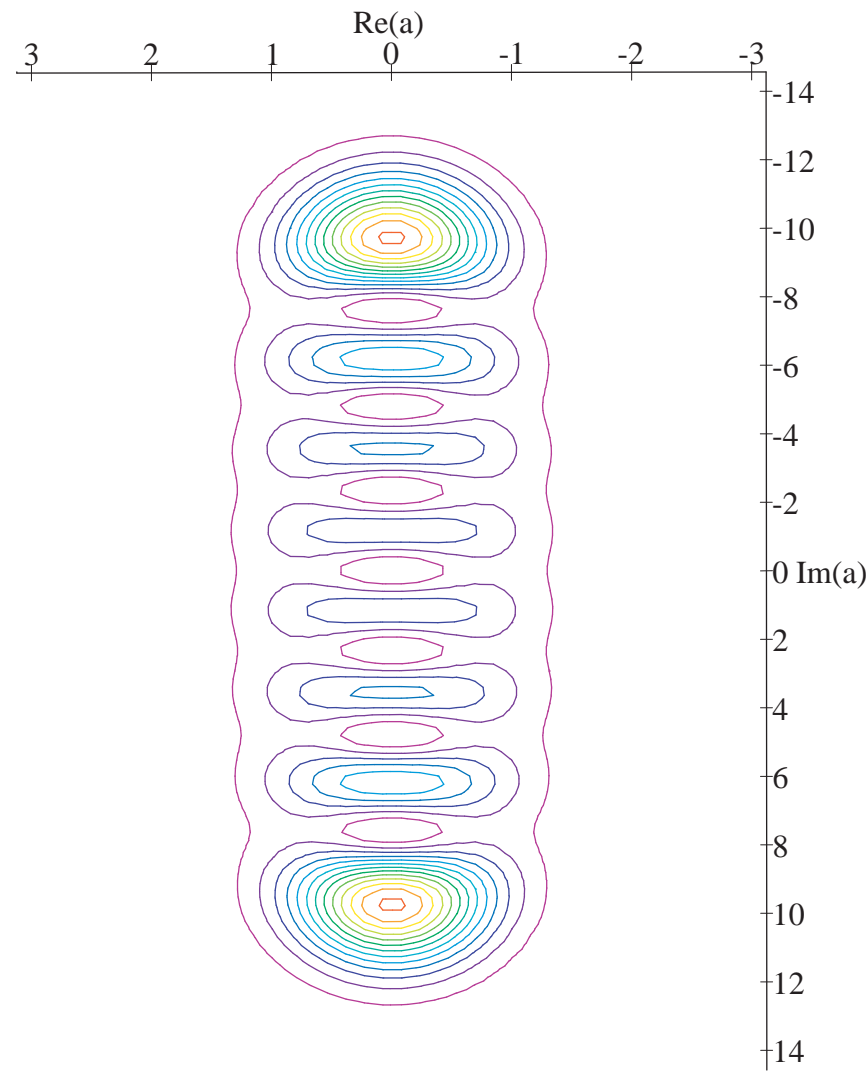

Figure 7: Contour graphic of the $Q(\alpha)$ function of the $m=7$ squeezed number state with $r=1.40$ 


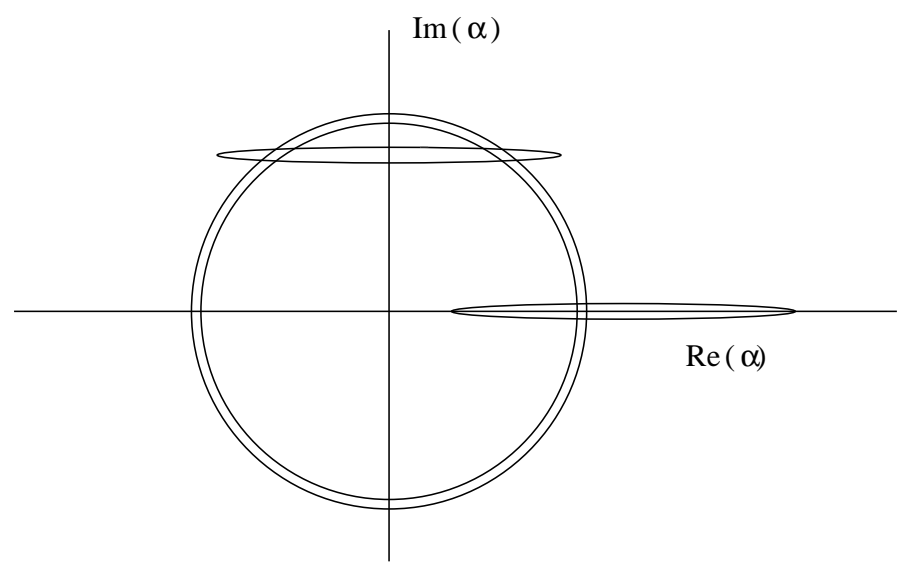

Figure 8: Phase space areas for: a number state $\mid m>$ (circular ring), a two photon coherent states $S^{\dagger} \mid \alpha>$ with real $\alpha$ ( right bottom ellipse) and a two photon coherent states with imaginary $\alpha$ (center top ellipse)
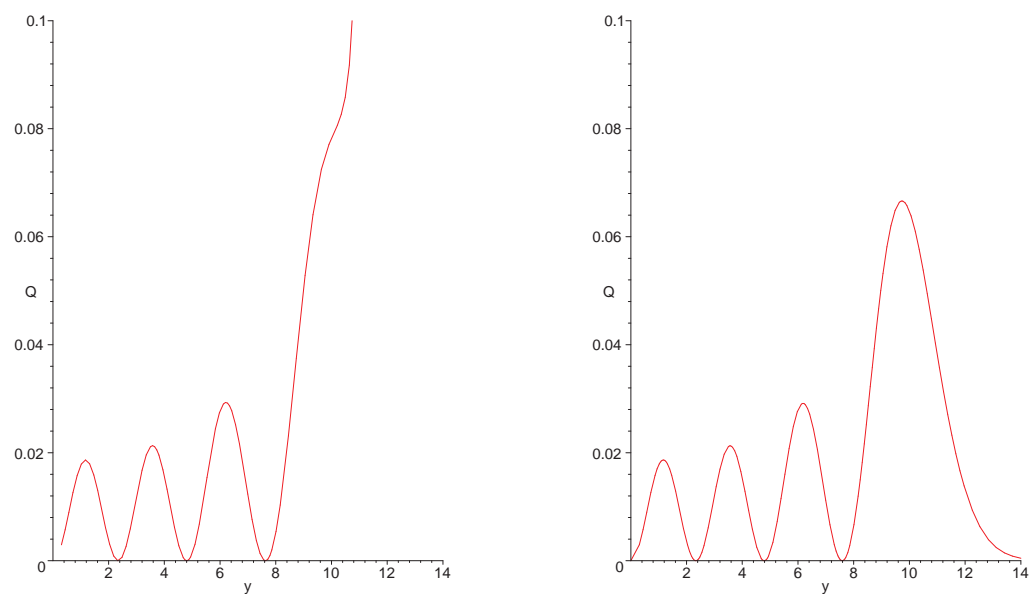

Figure 9: $\quad Q(\alpha=i y)$ function for the $m=7, r=1.4$ squeezed number state: The two areas overlapping approximation ( left) and the exact calculation ( right). 